\title{
Potential benefit of non-cardiac arrested thromboembolectomy of acute pulmonary embolism for the impaired right ventricular function
}

\author{
Masaho Okada $^{1}$ (D) $\cdot$ Hirotaka Watanuki $^{1} \cdot$ Kayo Sugiyama $^{1} \cdot$ Yasuhiro Futamura $^{1} \cdot$ Katsuhiko Matsuyama $^{1}$
}

Received: 10 June 2019 / Accepted: 26 September 2019 / Published online: 8 October 2019

(c) The Author(s) 2019

\begin{abstract}
Objectives Acute pulmonary thromboembolism is a fatal condition with high mortality rate in patients with hemodynamic collapse. Early diagnosis and aggressive treatment after circulatory collapse due to pulmonary thromboembolism are important. Although catheter-directed therapy or surgical thromboembolectomy could be considered, the prognosis of such cases is poor.

Methods We reviewed nine consecutive patients who underwent surgical intervention for acute pulmonary thromboembolism requiring cardiopulmonary resuscitation from March 2010 to May 2017. The patients' demographics, risk factors, operative and postoperative outcomes were retrospectively analyzed. All nine patients who experienced cardiopulmonary arrest or pulseless electrical activity had received cardiopulmonary resuscitation, including endotracheal intubation with cardiac massage. Computed tomography revealed massive pulmonary thromboembolism. Percutaneous cardiopulmonary support was initiated in six cases. Pulmonary thromboembolectomy was performed on a beating heart without aortic crossclamping in all patients

Results The mean operation time was $251.9 \pm 74.7 \mathrm{~min}$. Among the six patients who received preoperative percutaneous cardiopulmonary support, three were able to wean off cardiopulmonary bypass during the surgery. The other three cases were able to wean off within 3 days postoperatively. The mean postoperative intubation time was $56.3 \mathrm{~h}$. No mortality was observed. All patients were discharged with New York Heart Association functional class I without any neurological complications. No patients developed major and residual thrombi in the pulmonary artery upon postoperative computed tomography. Conclusions Pulmonary thromboembolectomy on a beating heart for acute pulmonary thromboembolism may be beneficial for minimizing right ventricular damage and improving clinical outcomes.
\end{abstract}

Keywords Acute massive pulmonary embolism · Thromboembolectomy · Cardiopulmonary resuscitation · Aortic crossclamping $\cdot$ Right ventricular dysfunction

\section{Introduction}

Acute pulmonary thromboembolism (PTE) is a fatal condition with a high mortality rate in patients with hemodynamic collapse [1]. Early diagnosis and aggressive treatment after circulatory collapse due to PTE are important for a good prognosis. Although catheter-directed therapy or surgical

Masaho Okada

okmasaho123@gmail.com

1 Department of Cardiac Surgery, Aichi Medical University School of Medicine, 1-1 Yazakokarimata, Nagakute, Aichi, Japan embolectomy can be considered in such cases, the prognosis had been very poor [2]. Here, we reported clinical cases of surgical thromboembolectomy for acute PTE requiring cardiopulmonary resuscitation (CPR).

\section{Methods}

We reviewed nine consecutive patients who underwent surgical intervention for acute PTE requiring CPR from March 2010 to May 2017. In the present study, massive PTE was defined as hemodynamic instability requiring heart massage for more than 5 min owing to persistent or repeated cardiac arrest. There were no cases 
regarding the acute on chronic status of chronic thromboembolic pulmonary hypertension. The mean duration of CPR was 16.7 min (range, 5-40 min). Percutaneous cardiopulmonary support (PCPS) was initiated in six with collapse-type PTE while continuing heart massage. Three intubated patients with non-collapse type PTE in whom heartbeat was fortunately restored with massive inotropic support were immediately transferred to the operating room for cardiopulmonary bypass. All patients were assessed for status of consciousness, the recovery of which was confirmed before surgical intervention. Patients who had severe brain damage received medical treatment without surgery. The patients' demographics, risk factors, and operative and postoperative outcomes were retrospectively analyzed (Table 1 ). The average age of the patients was 64 years (range, $48-81$ years); 8 were women. The risk factors for deep vein thrombosis (DVT) included prolonged bed rest in four cases, prolonged bed rest and chemotherapy in one case, oral medication in one case, and were unknown in the remaining three cases. No other risk factors of DVT, such as thrombotic diathesis, obesity, post-operative case, or mental disorder, were observed. The onset was in the hospital in one case and out of the hospital in the remaining eight cases. Echocardiography in all patients showed a severely dilated and remarkably hypokinetic right ventricle with a collapsed left ventricle. Computed tomography (CT) revealed massive PTE (Fig. 1).

\section{Surgical technique}

All operations were performed through a median sternotomy. Cardiopulmonary bypass (CPB) was established through the ascending aorta and by bicaval cannulation. Pulmonary thromboembolectomy was performed on a beating heart without aortic cross-clamping in all patients. A longitudinal incision was made on the left and right pulmonary

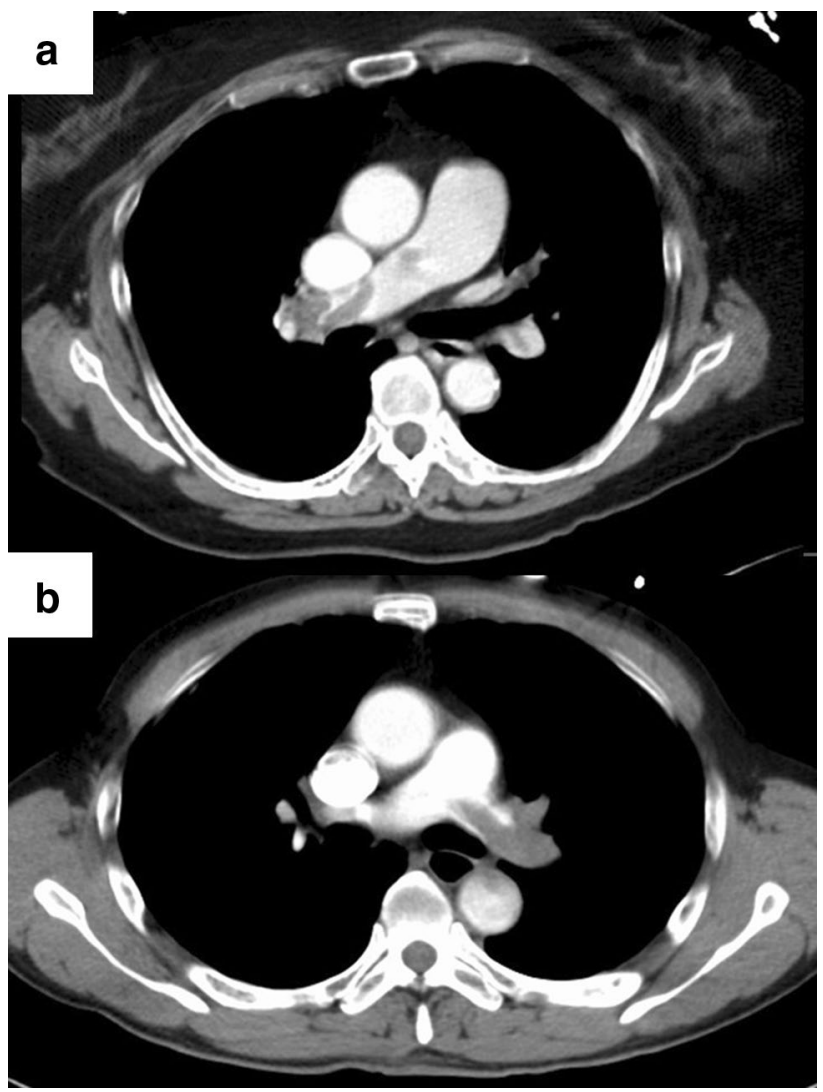

Fig. 1 Preoperative computed tomography scan shows a massive pulmonary embolus in the bilateral pulmonary artery

arteries, followed by clot removal using forceps under direct vision without endoscopy. The clots unidentified through direct vision were gently suctioned using a tube with dull and soft tip.
Table 1 Preoperative patient backgrounds

\begin{tabular}{llllllll}
\hline No. & Sex & Age & Risk factors & On set & CPA $(\mathrm{min})$ & $\begin{array}{c}\text { Preopera- } \\
\text { tive PCPS }\end{array}$ & D-dimer $(\mu \mathrm{g} / \mathrm{mL})$ \\
\hline 1 & F & 49 & Taking pills & Out-of-hospital & 15 & Yes & 177 \\
2 & F & 48 & Prolonged bed rest & Out-of-hospital & 35 & Yes & 161 \\
3 & F & 68 & - & Out-of-hospital & 40 & Yes & 210 \\
4 & F & 78 & - & Out-of-hospital & 5 & No & None \\
5 & F & 81 & Prolonged bed rest & Out-of-hospital & 8 & No & None \\
6 & F & 71 & Prolonged bed rest & Out-of-hospital & 10 & Yes & 182 \\
7 & M & 65 & Prolonged bed rest & In-hospital & 15 & Yes & 130 \\
8 & F & 55 & - & Out-of-hospital & 15 & Yes & 111 \\
9 & F & 62 & Prolonged bed rest & Out-of-hospital & 7 & No & 150 \\
& & & chemotherapy & & & & \\
\hline
\end{tabular}

$P C P S$ percutaneous cardiopulmonary support, $C P R$ cardiopulmonary resuscitation 


\section{Results}

The mean operation time was $251.9 \pm 74.7$ min (range, 168-423 min) (Table 2). Among the six cases that received preoperative PCPS, three were weaned off CPB during the surgery. The other three cases were weaned off PCPS at $12 \mathrm{~h}, 1$ day, and 3 days, respectively, after surgery. The mean postoperative intubation duration was $56.3 \mathrm{~h}$ (Table 2). One case developed transfusion-related acute lung injury as a postoperative complication and required 8 days of ventilator support. No mortality was observed in all cases. Echography confirmed that none of the cases developed DVT immediately after surgery, with subsequent control under heparin and warfarin anticoagulation. None of the cases developed major and residual thrombi in the pulmonary arteries on postoperative CT (Fig. 2). In all patients, postoperative echocardiography findings indicated no pulmonary hypertension (estimated right ventricular [RV] pressure $<30 \mathrm{mmHg}$ ) and a normalized RV ejection fraction. All patients were discharged with New York Heart Association functional class I without any neurologic complications.

\section{Discussion}

Primary diagnosis and aggressive treatment of acute PTE requiring CPR are critical for good prognosis [3]. Although catheter-directed therapy or surgical embolectomy can be considered in such cases, the prognosis had been very poor [2]. According to a systematic review by Loyalka et al., the mortality rates in acute PTE with CPR had remained considerably high at $52 \%$ after catheter-directed therapy and $46.3 \%$ after surgical therapy [4]. Keeling et al. reported a $32.1 \%$ mortality rate for patients who received surgical therapy [5], and the Japanese Society for Cardiothoracic Surgery also reported a high mortality rate (43.8\%) [6]. Moreover, a poor prognosis can be expected in cases complicated by

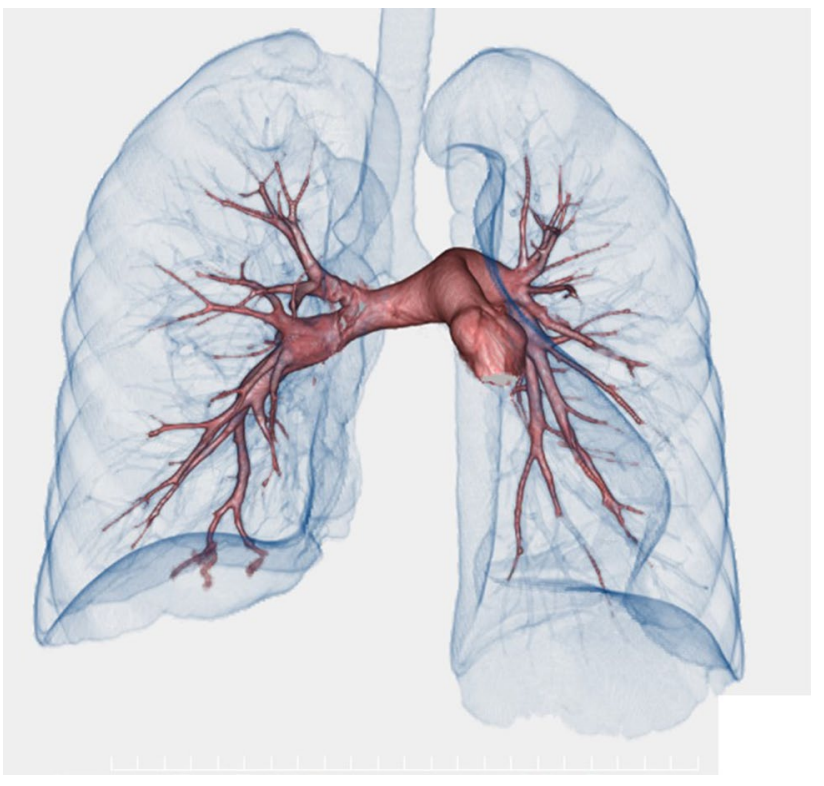

Fig. 2 Postoperative computed tomography scan shows the disappearance of the major thrombi in the bilateral pulmonary arteries

brain damage from hypoxia after CPR. Therefore, in this study, recovery of consciousness, with or without PCPS support, was assessed before surgical intervention; patients with brain damage were deemed to have no surgical indications. Early PCPS initiation and decision-making for surgery or relief of pulmonary hypertension/RV distension and appropriate clearance of pulmonary artery circulation without pulmonary hypertension should be considered [7]. Especially, the prevention of RV distention and dysfunction due to acute PTE is important for patients' recovery. Surgical thromboembolectomy in the acute phase of PTE is relatively easy given, that a fresh thrombus can be continuously connected and removed en bloc. On the other hand, performing thromboembolectomy on a beating heart or during cardiac arrest by aortic cross-clamping had been controversial. For better surgical view and complete removal of thrombi, the
Table 2 Perioperative and postoperative outcomes

\begin{tabular}{lllllll}
\hline No. & $\begin{array}{l}\text { Operative dura- } \\
\text { tion (min) }\end{array}$ & $\begin{array}{l}\text { Postoperative circu- } \\
\text { latory support }\end{array}$ & $\begin{array}{l}\text { Time to wean } \\
\text { off }(\mathrm{h})\end{array}$ & $\begin{array}{l}\text { Intubation } \\
\text { time }(\mathrm{h})\end{array}$ & $\begin{array}{l}\text { Hospital stay } \\
\text { (days) }\end{array}$ & Complication \\
\hline 1 & 292 & - & - & 12 & 13 & - \\
2 & 168 & PCPS, IABP & 24 & 24 & 39 & - \\
3 & 210 & - & - & 12 & 19 & - \\
4 & 228 & - & - & 18 & 20 & - \\
5 & 204 & PCPS, IABP & 12 & 192 & 44 & - \\
6 & 230 & - & - & 10 & 21 & - \\
7 & 228 & - & - & 11 & 39 & - \\
8 & 423 & PCPS & 72 & 192 & 78 & TRALI \\
9 & 284 & - & - & 36 & 32 & - \\
\hline
\end{tabular}

$I A B P$ intra-aortic balloon pumping, TRALI transfusion-related acute lung injury 
thromboembolectomy with cardiac arrest by aortic crossclamping has been preferable. In acute thromboembolism in different from chronic phase, pulmonary hypertension could be dramatically improved with the removal of major thrombus at bilateral pulmonary arteries. The clots remaining in a branch of the pulmonary artery can be dissolved by postoperative anticoagulant treatment $[3,8]$. In fact, in the current study, the clots in the peripheral pulmonary artery almost completely disappeared on postoperative CT. Moreover, in all patients, postoperative echocardiography findings indicated no pulmonary hypertension and a normalized RV ejection fraction.

The reason for difficulty in PCPS weaning is persistence of preoperatively severe RV dysfunction after surgery. The requirement of postoperative circulatory support, including PCPS and IABP, depends on the severity of preoperative RV damage. Therefore, patients with CPA, even for less than $10 \mathrm{~min}$, could receive postoperative circulatory support. However, preoperatively, cases of acute massive PTE showed severely deteriorated RV function, with RV dysfunction persisting after surgery. Accordingly, myocardial ischemia by aortic clamping could cause extreme damage to the right ventricle. Consequently, weaning off $\mathrm{CPB}$ could be challenging, and the mortality rates may increase $[5,9]$. In fact, a Japanese database demonstrated that longer CPB and aortic clamping led to poor prognosis [6]. Considering that operative procedures performed on a beating heart may minimize right ventricular dysfunction, these may allow smooth weaning off $\mathrm{CPB}$. After performing pulmonary thromboembolectomy on a beating heart in seven of eight patients who required CPR due to hemodynamic collapse after massive $\mathrm{PE}$, Konstantinov et al. reported that five cases survived [9]. In the current study, the ratio of patients who were weaned off CPB to those who were not weaned off CPB during the operation was $50 \%$; the cases that were not weaned off the CPB intraoperatively were weaned off PCPS within 3 days postoperatively. As a result, no mortality was observed in all cases.

In cases that undergo surgery on a beating heart, injury to a peripheral branch of the pulmonary artery can be a possibility. In their study, Konstantinov et al. used Fogarty catheters to remove clots in the peripheral pulmonary artery [9]. In our cases, the clots not confirmed on direct vision were gently suctioned using a tube with dull and soft tip. Extreme care should be taken to prevent injury to a peripheral pulmonary artery.

We believe that pulmonary thromboembolectomy on a beating heart for acute PTE may be beneficial in minimizing the right ventricular damage and improving clinical outcomes. However, if it is not sufficient or if there are subacute or chronic thrombi present, a more meticulous or careful thromboembolectomy should be performed with at least cardiac arrest to completely clear pulmonary circulation. Particularly, in cases with an acute on chronic status of chronic thromboembolic pulmonary hypertension, pulmonary endarterectomy must be attempted under deep hypothermic circulatory arrest to prevent serious residual pulmonary hypertension, which is sometimes lethal.

\section{Compliance with ethical standards}

Conflict of interest All authors declare that they have no conflict of interest.

Open Access This article is distributed under the terms of the Creative Commons Attribution 4.0 International License (http://creativecommons.org/licenses/by/4.0/), which permits unrestricted use, distribution, and reproduction in any medium, provided you give appropriate credit to the original author(s) and the source, provide a link to the Creative Commons license, and indicate if changes were made.

\section{References}

1. Dauphine C, Omari B. Pulmonary embolectomy for acute massive pulmonary embolism. Ann Thorac Surg. 2005;79:1240-4.

2. Konstantinides SV. 2014 ESC guidelines on the diagnosis and management of acute pulmonary embolism. Eur Heart $\mathrm{J}$. 2014;35:3145-6.

3. Matsuyama K, Nakayama T, Hagiwara H. Acute massive pulmonary thromboembolism with cardiac arrest. Kyobu Geka. 2011;64:807-11.

4. Loyalka P, et al. Surgical pulmonary embolectomy and catheterbased therapies for acute pulmonary embolism: a contemporary systematic review. J Thorac Cardiovasc Surg. 2018;156:2155-67.

5. Keeling WB, et al. Outcomes after surgical pulmonary embolectomy for acute pulmonary embolus: a multi-institutional study. Ann Thorac Surg. 2016;102:1498-502.

6. Minakawa $\mathrm{M}$, et al. Outcomes of pulmonary embolectomy for acute pulmonary embolism. Circ J. 2018;82:2184-90.

7. Munakata R, et al. Massive pulmonary embolism requiring extracorporeal life support treated with catheter-based interventions. Int Heart J. 2012;53:370-4.

8. Sadaba JR, et al. The surgical option in the management of acute pulmonary embolism. J Card Surg. 2008;23:729-32.

9. Konstantinov IE, et al. Acute massive pulmonary embolism with cardiopulmonary resuscitation: management and results. Tex Heart Inst J. 2007;34:41-5 (Discussion 45-46).

Publisher's Note Springer Nature remains neutral with regard to jurisdictional claims in published maps and institutional affiliations. 